\title{
Auditoría del proceso de dirección estratégica El caso del hotel Villa la Granjita de la ciudad de Santa Clara
}

\author{
Dayana Duffus Miranda* \\ Universidad Central Marta Abreu de Las Villas \\ Ana Beatriz Valiente Arbolaez \\ Geocuba VC-SS Grupo Empresarial \\ Mayelín García Peart \\ Boutique Varadero
}

\section{Resumen}

La hostelería en la industria turística se encuentra inmersa en un entorno turbulento donde, cada vez más rápido, los objetivos y estrategias pierden su sentido, de ahí que, para la gestión moderna, la calidad de las decisiones estratégicas se convierta en una meta impostergable. La administración se vale de disímiles métodos de dirección encaminados a una correcta planificación, una adecuada organización y al control de cada elemento de decisión. Cómo controlar la efectividad de este proceso (que garantice la toma de decisiones más eficaces) continúa siendo tema de discusión entre académicos y profesionales de este campo del conocimiento. Este artículo devela el papel de la auditoría de gestión como un instrumento útil para que las instalaciones hoteleras alcancen un comportamiento eficaz en el manejo de sus recursos y capacidades. El objetivo es auditar el proceso de dirección estratégica en su fase de análisis estratégico en el hotel Villa la Granjita, de la ciudad de Santa Clara (Cuba). Se estudian las características esenciales de la auditoría de gestión, su relación con el logro de la eficiencia y la eficacia y se proponen los pasos específicos para desarrollar este método de control en instalaciones hoteleras. La investigación brinda al hotel una herramienta que permite evaluar sistemáticamente la eficacia de un proceso medular para el mismo: el análisis estratégico.

\section{Palabras clave}

Auditoría de gestión, hoteles, eficiencia y eficacia, análisis estratégico.

Recibido: 17/11/2013 - Aceptado: 05/03/2014

*Correo electrónico:dduffusm@uclv.edu.cu·avaliente@vcl.geocuba.cu·mayelin@correoscuba.cu 


\title{
Strategic Management Audit: the case of the Villa la Granjita hotel in Santa Clara
}

\author{
Dayana Duffus Miranda* \\ Universidad Central Marta Abreu de Las Villas \\ Ana Beatriz Valiente Arbolaez \\ Geocuba VC-SS Grupo Empresarial \\ Mayelín García Peart \\ Boutique Varadero
}

\begin{abstract}
Hospitality in the tourism industry is immersed in a turbulent context in which goals and strategies loose meaningfulness very rapidly and therefore make the quality of strategic decision making an urgent goal in modern management. Management uses many dissimilar methods that focus on correct planning, on an adequate organization and on control of every decision element. How to control the effectiveness of his process (to warrant the most effective decision making) continues to be a discussion subject between academics and professionals in this knowledge field.

This paper clarifies the role of Management Audit as a useful instrument for hotels to reach efficiency in the management of their resources and capabilities. The goal is to audit the Strategic Management Process in the Strategic Analysis Phase in the "Villa la Granjita” hotel in Santa Clara, Cuba. The essential characteristics of Management Audit as well as it's relationship with the achievement of efficiency and efficacy are studied, and specific steps to develop this control method in the hotel sector are proposed. This research offers hotels a tool that allows the systemic evaluation of a key process: strategic analysis.
\end{abstract}

\section{KeY WORDS}

Management Audit, hotels, efficiency and efficacy, strategic analysis.

*E-mail:dduffusm@uclv.edu.cu·avaliente@vcl.geocuba.cu·mayelin@correoscuba.cu 


\section{Introducción}

La dinámica y las tendencias actuales del sector turístico en el mundo son verdaderamente inciertas, más aún con las crecientes exigencias de los consumidores. Cuba no es ajena a esta situación si se considera que el turismo en la isla se ha convertido en una actividad económica fundamental que busca una oferta turística competitiva y sustentable, pero que no ha dejado de tener desaciertos en la toma de decisiones. Por ello es indispensable una continua mejora del proceso de dirección estratégica en las diferentes instalaciones del sector, apoyada en la inevitable urgencia de planificar, expresada en los lineamientos que rigen la política económica del país. Sin embargo, para planificar de manera correcta deben distinguirse los errores que se cometen en la actualidad, no solo en las operaciones contables sino también en la gestión de procesos. Así, se torna imprescindible realizar un análisis sistemático de la administración y utilización de los recursos mediante el cumplimiento de objetivos y metas, así como una evaluación de la economía, eficiencia y eficacia en su utilización. En este contexto, el análisis estratégico juega un papel trascendental dentro de la planificación, al aportar las bases requeridas para una correcta toma de decisiones. En consecuencia, auditar continuamente esta fase de análisis estratégico (en busca de posibles deficiencias) es la razón primaria de las auditorías de gestión, que permiten reconocer de forma constante y previsora los errores cometidos y las opciones de actuación en el futuro.

El proceso de dirección estratégica es capaz de llevar al sector turístico a una proyección que garantice su desarrollo sostenible en el largo plazo, de ahí que todo esfuerzo encaminado a resolver la necesidad de evaluar de manera sistemática este proceso es un avance en favor de un desempeño eficaz del sector. Por ello, el objetivo general de esta investigación es efectuar una auditoría de gestión al proceso de dirección estratégica en su fase de análisis estratégico en el hotel Villa la Granjita.

Se parte de una secuencia metodológica deductiva en la cual se realiza una revisión bibliográfica sobre aquellas áreas de estudio afines con el tema a explorar; luego se valoran todos los textos adquiridos para identificar secuencias de análisis comunes y posibles vacíos en el conocimiento, lo cual reafirma la necesidad de aplicar un instrumento que permita el diagnóstico sistemático de la función de dirección estratégica, para tal fin se utilizó una secuencia 
metodológica inductiva, por medio de técnicas de investigación cuantitativas y cualitativas, fundamentalmente el cuestionario, la observación directa y el análisis de documentos.

\section{Antecedentes del estudio: la auditoría de gestión como método indispensable en el logro de la eficiencia y la eficacia en el sector hotelero}

La auditoría de gestión es joven como ciencia y es resultado del desarrollo social, del avance tecnológico y de la práctica productiva. No se trata de un simple análisis económico, sino de un examen mucho más profundo, pues no se limita a mostrar cifras o explicaciones. La auditoría de gestión debe comprobar cada elemento y poner al descubierto las prácticas antieconómicas, ineficientes e ineficaces, detallando las causas y condiciones que las provocan y el efecto que producen (López y García, 2007: 8).

La denominación auditoría de gestión funde en una dos clasificaciones tradicionales: auditoría administrativa y auditoría operacional. Así, es posible afirmar que auditoría de gestión es el examen crítico, sistemático y detallado de las áreas y controles operacionales de un ente, realizado con independencia y mediante técnicas específicas, con el propósito de emitir un informe profesional sobre la eficacia, la eficiencia y la economicidad en el manejo de los recursos y orientado a que la toma de decisiones sirvan para mejorar la productividad del mismo.

Su objetivo primordial es descubrir deficiencias o irregularidades en ciertas partes de la empresa y apuntar sus posibles remedios, con la finalidad de ayudar a la dirección a lograr una administración más eficaz. Su propósito es examinar y valorar los métodos y el desempeño en todas las áreas. Los factores de la evaluación abarcan el panorama económico, el manejo del personal y la utilización del equipo y de los sistemas (Palominos, 2006: 5).

Se puede realizar una auditoría de gestión a una función específica, a un departamento o grupo de departamentos, a una división o grupo de divisiones o a la empresa en su totalidad; algunas auditorías abarcan una combinación de dos o más áreas. De forma general, una auditoría de gestión debe ser económica, preventiva, ágil, pertinente, crítica, sistemática, analítica y objetiva (Palominos, 2006: 7). 
En la actualidad, a medida que el entorno de las actividades económicas se transforma, estas se enfrentan a un cambio progresivo y acelerado, por lo que no es suficiente contar solo con una evaluación de sus estados financieros (que es el análisis de la situación pasada), sino que se requiere una visión hacia el futuro, con un enfoque concentrado en la evaluación de las actividades que giran en torno al cumplimiento de las metas y los objetivos trazados por las organizaciones.

La auditoría de gestión es fundamental ante esta situación, por cuanto permite medir las actuaciones pasadas y presentes de la administración, para mejorar el futuro, corregir sus errores y elevar el grado de economía, eficiencia y eficacia en sus operaciones y actividades.

Cuando hablamos de eficiencia hacemos alusión a la capacidad de reducir al mínimo los recursos usados para alcanzar los objetivos de la organización, y cuando nos referimos a la eficacia hablamos inexcusablemente de la capacidad para determinar los objetivos apropiados: hacer lo que se debe hacer (Stoner, Freeman y Gilbert, 2006: 13-14).

La actividad turística en Cuba presenta una serie de brechas que no le permiten lograr un desarrollo totalmente eficiente, por lo que requiere aumentar sus indicadores de eficiencia incrementando el nivel ocupacional de la infraestructura hotelera, mejorando la calidad de los servicios turísticos, elevando la eficacia en el proceso de construcción, en el desarrollo armónico de la infraestructura extrahotelera, en la reparación y remodelación de hoteles, así como en la sustitución de importaciones y en la profundización de la promoción internacional (Pérez, 2011: 14).

Más que dirigir habrá que liderar, en vez de vender productos o servicios habrá que satisfacer expectativas, y los precios serán aquellos que estén dispuestos a pagar los consumidores, quienes cada vez tendrán más información y cultura del valor (Gallego, 2007: 30). Para insertarse con éxito en esta realidad es indispensable auxiliarse de las auditorías de gestión sistemáticas, que permitan reconocer de forma constante y previsora los errores cometidos y las posibles formas de actuación en el futuro. Así, los encargados del proceso directivo podrán comprender mejor qué elementos carecen de importancia, cuáles han quedado envueltos en costumbres y maneras ya obsoletas, cuáles deben ser cambiados, corregidos, mejorados o simplemente eliminados; dónde está la clave del éxito, cómo obtener un servicio más eficiente sin perder de vista las 
costumbres y los deseos de los clientes, de forma que nuestros hoteles logren ser eficaces y competitivos, del agrado del mercado internacional.

\section{Metodología para efectuar la auditoría de gestión en el proceso de dirección estratégica}

La auditoría de gestión como una herramienta fundamental del control determina las deficiencias de las organizaciones en su administración. Al efectuar este tipo de evaluación no puede perderse de vista que la empresa se desenvuelve en un medio externo e interno; las evaluaciones tienen a la vez el carácter de diagnóstico y de pronóstico, y servirán de base para configurar las acciones venideras que se recomendarán a la empresa respecto a lo auditado. A fin de llevarla a cabo con éxito debe determinarse la metodología a seguir para evaluar con objetividad la gestión de la empresa, la función o el área objeto de estudio.

En cuanto a esta metodología, como en casi todos los procesos empresariales, existen diversas opiniones:

En el cuadro 1 aparecen las fases más generalizadas de las auditorías de gestión, todas las metodologías parten del supuesto de que la auditoría se realiza a todas las áreas de la empresa. Sin embargo, en el mundo empresarial actual la optimización de recursos, incluido el tiempo, es vital, por lo que es indispensable determinar cuál es el área crítica de la empresa para enfocar sobre ella el esfuerzo. La auditoría objeto de esta investigación será realizada a la fase de análisis estratégico del proceso de dirección estratégica en el hotel Villa la Granjita de Santa Clara, Cuba. Se llevará a cabo siguiendo un programa de nueve pasos fundamentales (figura 1), derivado de las metodologías descritas (cuadro 1) y adaptado a las necesidades de la investigación. El programa que se propone es flexible, con una consistencia lógica y racional, apropiada para el tiempo y los recursos disponibles, e implica la participación de los trabajadores y directivos del hotel. Por las características mencionadas consideramos que es aplicable a otros contextos económicos y sociales. 
Cuadro 1. Fases de las metodologías para el diagnóstico empresarial

\begin{tabular}{|c|c|c|c|c|}
\hline \multirow{2}{*}{ Metodología } & \multicolumn{4}{|c|}{ ETAPAS DE LA AUDITORÍA } \\
\hline & Planeación & $\begin{array}{l}\text { CONOCIMIENTO } \\
\text { GENERAL DE LA } \\
\text { ENTIDAD }\end{array}$ & $\begin{array}{l}\text { EJECUCIÓN DE } \\
\text { LA AUDITORÍA }\end{array}$ & $\begin{array}{l}\text { INFORME DE } \\
\text { AUDITORÍA }\end{array}$ \\
\hline $\begin{array}{l}\text { Metodología para el } \\
\text { diagnóstico tecnológico de } \\
\text { pymes (DT- pymes) (Instituto } \\
\text { Catalá de Tecnología,1993) }\end{array}$ & & $\mathrm{X}$ & $\mathrm{X}$ & $\mathrm{X}$ \\
\hline $\begin{array}{l}\text { Metodología propuesta por el } \\
\text { Programa de Autoevaluación } \\
\text { de Empresas (Cámara Oficial } \\
\text { de Comercio, Industria y } \\
\text { Navegación de Barcelona, } \\
\text { 1992) }\end{array}$ & & & $\mathrm{X}$ & $\mathrm{X}$ \\
\hline $\begin{array}{l}\text { Metodología para el } \\
\text { diagnóstico de la } \\
\text { comercialización de opciones } \\
\text { turísticas en agencias de } \\
\text { viajes receptivo-minoristas } \\
\text { (Morales, 2007) }\end{array}$ & & $\mathrm{X}$ & $\mathrm{X}$ & \\
\hline $\begin{array}{l}\text { Metodología propuesta para } \\
\text { el análisis del estado actual } \\
\text { del control de gestión en } \\
\text { hoteles (Prieto, 2007). }\end{array}$ & & $\mathrm{X}$ & $\mathrm{X}$ & $\mathrm{X}$ \\
\hline $\begin{array}{l}\text { Metodología para el } \\
\text { diagnóstico general con } \\
\text { vistas al perfeccionamiento } \\
\text { de la gestión en entidades } \\
\text { turísticas (Martínez et al., } \\
\text { 2007). }\end{array}$ & $\mathrm{X}$ & & $\mathrm{X}$ & $\mathrm{X}$ \\
\hline
\end{tabular}

Fuente: Elaboración propia. 


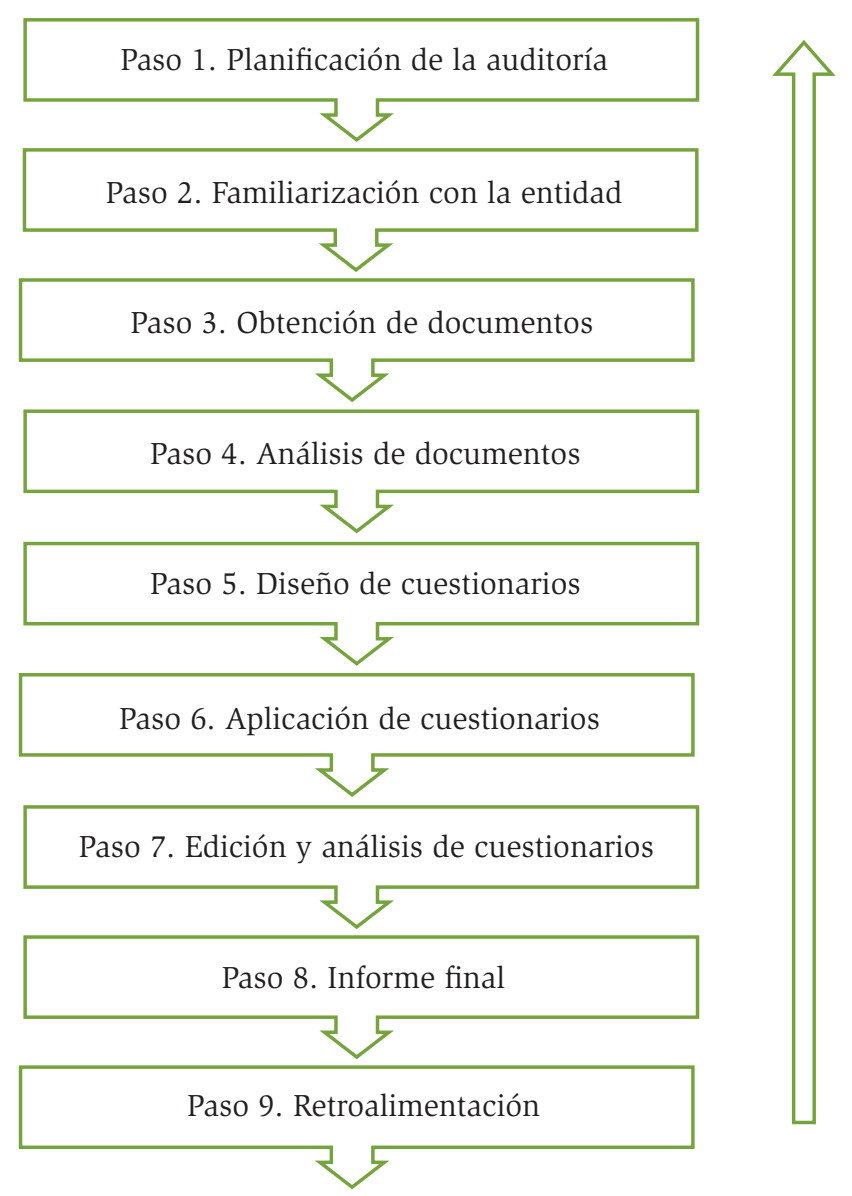

Fuente: Elaboración propia

Figura 1. Programa para la auditoría de gestión en el hotel Villa la Granjita, de Santa Clara, Cuba

\section{Discusión de los principales resultados}

Tras el diseño de la auditoría de gestión para el hotel Villa la Granjita expondremos los resultados obtenidos a partir de las técnicas de investigación empleadas, los cuales permiten ejercer el control sistemático de la fase de análisis estratégico del proceso de dirección estratégica, en busca de la eficiencia y la eficacia en la toma de decisiones. 
Cada dimensión parte del análisis estratégico de la empresa y se examina y califica mediante la siguiente escala (Heredia y Martínez, 2002: 12):

- Inadecuado: esta calificación es inaceptable para el desarrollo del modelo de dirección estratégica en hoteles.

- Aceptable: evaluación aún por debajo de los requerimientos para desarrollar de manera efectiva la dirección estratégica en hoteles.

- Promedio: esta valoración puede ser aceptada temporalmente, mas debe ser mejorada.

- Bueno: esta calificación, desarrollada por encima del promedio, se determina cuando satisface los requerimientos para efectuar la dirección estratégica, y deja muy pocas cosas importantes por mejorar o perfeccionar.

- Excelente: es un estándar que evidencia el aspecto evaluado como efectivo y eficiente para la dirección estratégica.

Es pertinente aclarar que los porcentajes pueden sumar más de $100 \%$, ya que en las encuestas tanto a empleados como a directivos se utilizan preguntas de múltiple elección. Este es el caso de la preguntas referidas en la dimensión 3: a los participantes en el análisis del diagnóstico externo, fuentes de obtención de información para el diagnóstico externo; fuentes de obtención de información sobre proveedores y distribuidores así como clientes. En la dimensión 4 se usan también este tipo de preguntas para determinar a los participantes en la elaboración del diagnóstico interno, los puntos clave del éxito y los elementos que definen las ventajas competitivas de la empresa. Por último, en la dimensión 5, los encuestados pueden seleccionar varias opciones a la vez en la pregunta referida a los participantes en la elaboración de los objetivos por unidad.

\section{Elaboración de la misión}

Esta dimensión se compone de tres variables.

\section{CONTENIDO DE LA MISIÓN}

La misión de la empresa presenta una redacción acorde con los requisitos indispensables, en ella se expresan las necesidades del mercado (las cuales son atendidas), los mercados que se satisfacen, la filosofía empresarial con que trabajan sus empleados y sus ventajas competitivas para captar la preferencia 
de los clientes; con todo, aunque la promoción es un elemento importante no es lo suficientemente trascendente para considerarla una ventaja competitiva a desarrollar por la instalación, pues debe comenzarse por poseer un servicio eficiente y eficaz para después ser objeto de promoción. Es menester señalar que la misión no contempla el tipo de hotel de la instalación. La calificación otorgada al contenido de la misión es: bueno.

\section{CARACTERÍSTICAS DE SU DISEÑO}

La misión está redactada en términos amplios y abarcadores pero a la vez precisos, de modo que expresa el objeto social de la entidad.

Según los resultados mostrados en el cuadro 2 existe desconocimiento sobre quién redacta la misión de la empresa. Esto lleva a concluir que la misión no es analizada ni discutida por todos los trabajadores. La calificación otorgada al diseño de la misión es: promedio.

Cuadro 2. Participantes que intervienen en la redacción de la misión

\begin{tabular}{lcc} 
Participantes & Directivos (\%) & Trabajadores (\%) \\
\hline La dirección & 50 & 20 \\
Colectivo de trabajadores & 20 & 50 \\
Colectivo de trabajadores guiados por & 30 & 10 \\
la dirección & - & 20 \\
No conoce quién la redacta & - & \\
\hline
\end{tabular}

Fuente: Elaboración propia.

\section{INSTRUMENTO MOVILIZADOR Y ESCLARECEDOR DEL TRABAJO}

De acuerdo con los datos del cuadro 3 y el análisis documental de los objetivos empresariales, estos últimos sí tributan al cumplimiento de la misión de la empresa debido a que están dirigidos fundamentalmente a perfeccionar los métodos y estilos de dirección, con una mayor y efectiva participación de los trabajadores en la toma de decisiones y en el mejoramiento continuo del producto turístico que comercializan; además de apoyarse en el aumento de la gestión de la promoción y comercialización de los productos y servicios y en la elevación del grado de satisfacción de los clientes, a partir del fortalecimiento de la gestión de calidad. 
Cuadro 3. Medida en que los objetivos tributan al cumplimiento de la misión

\begin{tabular}{lcc} 
Medida & Directivos (\%) & Trabajadores (\%) \\
En gran medida & 20 & 40 \\
Totalmente & 80 & 60 \\
\hline
\end{tabular}

Fuente: Elaboración propia.

Mediante la observación directa se comprobó que la misión es comunicada a todos los trabajadores, al encontrarse en un lugar visible y de tránsito obligatorio de quienes trabajan o visitan el hotel. La misión presenta cierta estabilidad en el tiempo, a pesar de que sufrió un pequeño cambio cuando el hotel se volvió parte de la empresa Hotel Los Caneyes, pero sigue manteniendo sus elementos esenciales. La calificación obtenida es: excelente.

La dimensión referida a la misión fue calificada como bueno, al tener pocas cosas importantes que mejorar en cuanto al contenido y al considerar su diseño como promedio; a pesar de que es excelente como instrumento movilizador y esclarecedor del trabajo.

\section{Confección de la visión}

Esta dimensión está compuesta por dos variables.

CONTENIDO DE LA VISIÓN

La visión de la empresa presenta una redacción coherente y refleja la necesidad del mercado que desean atender en el futuro, a cuáles mercados se dirigirán, el tipo de hotel que quieren ser, con qué capacidades procuran lograrlo y las ventajas competitivas que pretenden alcanzar y mantener en el largo plazo. La calificación otorgada por el contenido de la visión es: excelente.

CARACTERÍSTICAS DE SU DISEÑO

La visión está expresada en términos desafiantes pero a la vez realistas y viables. Tiene gran compatibilidad con la misión pues ambas están formuladas respecto a la perfección en los servicios de alojamiento, gastronomía y recreación, a partir del incremento del nivel de calidad del servicio y la profesionalidad. 
A partir de los cuadros 4 y 5 y de los resultados obtenidos de la revisión documental de la visión interrelacionada con los objetivos estratégicos, se verifica que estos últimos contribuyen al cumplimiento de la misma, esencialmente cuando se refieren al aumento de la gestión de calidad y la profesionalidad; uno de los aspectos relevantes tratados en la visión. De igual modo, se puede concluir que la visión es conocida por todos los empleados del hotel, a pesar de que desconocen quiénes participan en su redacción. La calificación otorgada al diseño de la visión es: bueno.

La confección de la visión es evaluada como bueno porque, a pesar de tener un contenido excelente, no es claro quiénes participan en el diseño (bueno).

Cuadro 4. Participantes que intervienen en la redacción de la visión

\begin{tabular}{lcc} 
Participantes & Directivos (\%) & Trabajadores (\%) \\
\hline La dirección & 50 & 20 \\
Colectivo de trabajadores & 20 & 50 \\
Colectivo de trabajadores guiados por la dirección & 30 & 10 \\
No conoce quién la redacta & - & 20 \\
\hline
\end{tabular}

Fuente: Elaboración propia.

Cuadro 5. Nivel de conocimiento de la visión por parte de los trabajadores

NIVEL

En gran medida

Totalmente

Fuente: Elaboración propia.
Trabajadores (\%)

20

80 


\section{Diagnóstico externo}

El diagnóstico externo será dividido en dos variables fundamentales.

CONTENido DEL Diagnóstico EXTERno

El análisis documental puso de manifiesto que cuando se ha realizado el diagnóstico externo de la empresa, en lo que se refiere al macroentorno solo se examina la variable demográfica en cuanto al crecimiento y a la capacidad habitacional. No obstante, el estudio del microentorno es más completo al considerar las cuatro variables básicas que lo componen: los clientes son estudiados por segmentos, basándose en su país de origen, sexo, edad, poder adquisitivo y preferencias. Los proveedores y distribuidores se clasifican en actuales y potenciales; se analizan los principales competidores en cuanto a la calidad en el servicio y los elementos de diferenciación. Debido a la falta de profundización en las variables observadas y al poco estudio del macroentorno; el contenido del diagnóstico externo fue calificado como inadecuado.

CARACTERÍsticas DE SU DiseÑo E IMPLEMENTACIÓN.

Al meditar sobre los resultados reflejados en los cuadros 6 a 9 y lo expuesto en los documentos, se dable concluir que no existe un consenso sobre quiénes elaboran el diagnóstico estratégico. La información para elaborar tal diagnóstico se obtiene de la Oficina Nacional de Estadística, del Ministerio de Turismo y de revistas especializadas en el tema, y la búsqueda de conocimientos sobre proveedores, clientes y distribuidores se realiza sobre todo mediante la encuesta y la observación directa.

Cuadro 6. Participantes en el análisis del diagnóstico externo

\begin{tabular}{lcc} 
Participantes & Directivos (\%) & TRABajadores (\%) \\
Trabajadores con conocimientos & - & 20 \\
$\begin{array}{l}\text { Administradores y trabajadores } \\
\text { conocimientos }\end{array}$ & 90 & 80 \\
\begin{tabular}{l} 
La Dirección \\
\hline
\end{tabular} & 10 & - \\
\hline
\end{tabular}

Fuente: Elaboración propia. 
Cuadro 7. Fuentes de información para el diagnóstico externo

\begin{tabular}{lc} 
FuEntes & Directivos (\%) \\
Oficina Nacional de Estadísticas & 40 \\
Ministerio de Turismo & 80 \\
Revistas especializadas & 70 \\
Servicios en bancos de datos & 30 \\
Consultorías & 20 \\
\hline
\end{tabular}

Fuente: Elaboración propia.

Cuadro 8. Fuentes de información sobre proveedores y distribuidores

\begin{tabular}{lc} 
FuENTES & Directivos (\%) \\
Indagación & 10 \\
Observación & 90 \\
Encuesta & 40 \\
\hline
\end{tabular}

Fuente: Elaboración propia.

Cuadro 9. Fuentes de información sobre clientes

\begin{tabular}{lc} 
FuENTES & Directivos (\%) \\
Indagación & 20 \\
Observación & 60 \\
Encuesta & 60 \\
\hline
\end{tabular}

Fuente: Elaboración propia.

Con los datos recolectados como punto de partida se identifican amenazas y oportunidades, pero no se utilizan en la elaboración de los posibles escenarios en que se podría desenvolver la empresa en el futuro, lo cual es de gran relevancia pues ayuda a planificar y proyectar mejor lo que después pudiera afectar el buen funcionamiento de la entidad.

Los resultados de este diagnóstico estratégico se tienen presentes al determinar los objetivos, aun cuando estos están cimentados sustancialmente en 
los Lineamientos de la Política Económica y Social del Partido y la Revolución aprobados en el VI Congreso del Partido Comunista de Cuba.

La calificación recibida por el diseño y la implementación del diagnóstico externo es: inadecuado. El diagnóstico externo como dimensión tiene esta calificación debido a que tanto su contenido como su diseño e implementación son inaceptables para el desarrollo del modelo de dirección estratégica.

\section{Dimensión 4: Diagnóstico interno}

El diagnóstico interno se audita teniendo en cuenta dos variables.

Contenido del diagnóstico interno.

El contenido de este diagnóstico es prácticamente nulo, se limita al diseño de un plan de acción para cada área funcional, pero sin haber realizado un inventario de los recursos humanos, tecnológicos, naturales e intangibles con que se cuenta ni de las capacidades de las que se dispone. Tampoco aparece reflejado algún análisis funcional por área que muestre las características positivas y negativas del funcionamiento de cada una y respalde las acciones por adoptar. Por lo anterior, el contenido del diagnóstico interno se considera inadecuado.

CARACTERÍSTICAS DE SU DISEÑO E IMPLEMENTACIÓN.

Al corroborar la información obtenida en el análisis de documentos y la expuesta en los cuadros 10 y 11, se evidencia que el diagnóstico interno es efectuado fundamentalmente por el equipo directivo. Se realiza una vez al año, aunque algunos directivos no conocen la periodicidad con que se lleva a cabo.

Cuadro 10. Participantes en la elaboración del diagnóstico interno

PARTICIPANTES

Administradores intermedios

Equipo directivo

Empleados
Directivos (\%)

50

90

40

Fuente: Elaboración propia. 
Cuadro 11. Frecuencia con que se realiza el diagnóstico interno

\section{FRECUENCIA}

Por trimestre

Anualmente

Fuente: Elaboración propia.

\section{DiRectivos (\%)}

10

90

A pesar de que los cuestionarios apuntan que las opiniones de clientes y trabajadores son tomadas en cuenta (cuadro 12), y en la documentación aparece la declaración de los puntos fuertes y débiles, no se observa perfil estratégico alguno, a pesar de que los directivos afirman su realización (cuadro 13); solo se incluyen las principales actividades que debe adoptar cada área funcional.

Cuadro 12. Grado en que las percepciones de clientes y empleados son tomadas en cuenta en el diagnóstico interno

\begin{tabular}{lcc} 
Nivel & Directivos (\%) & Trabajadores (\%) \\
Medianamente & 40 & - \\
En gran medida & 40 & 30 \\
Totalmente & 20 & 70 \\
\hline
\end{tabular}

Fuente: Elaboración propia.

Cuadro 13. Grado en que se realiza un perfil estratégico de la empresa

NIVEL

En poca medida

Medianamente

En gran medida

Totalmente
Directivos (\%)

20

20

20

40

Fuente: Elaboración propia. 
Según los cuadros 14 y 15, la clave del éxito del hotel está basada en la calidad del servicio a los empleados, la imagen y las diversas ofertas recreativas; $y$, en menor medida, en el efectivo servicio de reserva y las rutinas organizacionales de alojamiento. Para quienes elaboran el diagnóstico la reputación carece de importancia, a pesar de ser un elemento tan trascendente para atraer clientes potenciales, sobre todo en las instalaciones turísticas que tienen una competencia tan fuerte. Definen las ventajas competitivas a partir de comparaciones con otras instalaciones del mismo sector, fortalezas altamente valoradas por el sector turístico, y de estudios realizados por la propia entidad; las opiniones de los clientes son consideradas en menor medida. Aunque esta información se derivó de los cuestionarios, lo cierto es que no existen documentos que la respalden. Las características del diseño y la implementación del diagnóstico interno se evaluaron como inadecuadas.

\section{Cuadro 14. Puntos clave del éxito}

Puntos Clave

Directivos (\%)

Reputación

Imagen

70

Calidad del servicio de los empleados

80

Rutinas organizacionales de alojamiento 10

Efectivo servicio de reserva 20

Diversidad de ofertas recreativas 60

Fuente: Elaboración propia.

Cuadro 15. Elementos que definen las ventajas competitivas de la empresa

ELEMENTOS Directivos (\%)

Opiniones de los clientes 20

Comparaciones realizadas con otras instalaciones del mismo tipo 50

Fortalezas altamente valoradas por el sector turístico 50

Estudios realizados por la propia entidad 50

Fuente: Elaboración propia a partir de los cuestionarios aplicados. 
El diagnóstico interno fue evaluado como inadecuado pues su contenido, diseño e implementación son inaceptables para el logro de un análisis estratégico consistente.

\section{Objetivos estratégicos}

Los objetivos estratégicos se auditaron teniendo en cuenta dos variables fundamentales.

CONTENIDO DE LOS OBJETIVOS

Los objetivos tienen una redacción sencilla y acorde con los requisitos mínimos indispensables; quedan expresados los resultados deseados y que son elementales para el logro de la misión. Existe una correcta relación entre los objetivos operativos, sus indicadores y los valores por alcanzar. La calificación dada al contenido de los objetivos es: excelente.

CARACTERÍSTICAS DEL DISEÑo E IMPLEMENTACIÓN DE LOS OBJETIVOS

Teniendo en cuenta los resultados de los cuestionarios, descritos en los cuadros 16 y 17, es posible concluir que en la definición de los objetivos para cada unidad funcional casi siempre participan los trabajadores junto con los directivos. Además, estos objetivos son comunicados a todos y a cada uno se le asignan prioridades por nivel de importancia.

Cuadro 16. Participantes en la elaboración de los objetivos por unidad

\begin{tabular}{lcc} 
Participantes & Directivos (\%) & Trabajadores (\%) \\
Máximo responsable de cada departamento & 60 & 30 \\
Trabajadores & 80 & 100 \\
Directivos & 70 & 70 \\
\hline
\end{tabular}

Fuente: Elaboración propia.

Cuadro 17. Nivel de comunicación y asignación de prioridades a los objetivos

$$
\text { Nivel }
$$

En gran medida

Totalmente

Fuente: Elaboración propia.

$$
\text { Directivos (\%) }
$$$$
60
$$$$
40
$$

50 
Según las respuestas de los directivos (corroboradas en el análisis documental), los objetivos se encuentran correctamente respaldados por objetivos operativos y coordinados con el resto de los niveles organizacionales (cuadros 18 y 19).

Cuadro 18. Grado en que los objetivos estratégicos están respaldados por objetivos operativos

NiveL Directivos (\%)

En gran medida 40

Totalmente 60

Fuente: Elaboración propia.

Cuadro 19. Grado en que los objetivos están coordinados con el resto de los niveles

NiveL

En gran medida

Totalmente

Fuente: Elaboración propia.

Mediante la revisión documental se comprobó que los objetivos responden a lo expuesto en la misión y la visión. Son redactados con claridad, son factibles, realistas e implican desafío. Están orientados hacia resultados concretos, verificables, medibles, cuantificables y motivadores. El número de objetivos es manejable y presentan una adecuada relación con los indicadores y valores por alcanzar.

Los cuadros 20 y 21 ponen de manifiesto que existe retroalimentación oportuna a partir de la implantación de los objetivos, y que estos reflejan y requieren las principales actividades de los trabajadores. La calificación concedida por el diseño y la implementación de los objetivos es: excelente.

Los objetivos estratégicos tienen una calificación de excelente por cumplir tanto en su contenido como en el diseño e implementación con los parámetros establecidos; no obstante, debe señalarse que tienen un alto grado de dependencia de las dimensiones anteriores, lo cual hace que pierdan un poco de credibilidad al estar fundamentados en un diagnóstico inadecuado. 
Cuadro 20. Nivel de retroalimentación de los objetivos

\begin{tabular}{lcc} 
Nivel & Directivos (\%) & Trabajadores (\%) \\
En gran medida & 40 & 50 \\
Totalmente & 60 & 50 \\
\hline
\end{tabular}

Fuente: Elaboración propia.

Cuadro 21. Medida en que los objetivos requieren las actividades de los directivos y trabajadores

\begin{tabular}{lcc} 
Nivel & Directivos (\%) & Trabajadores (\%) \\
En gran medida & 60 & 40 \\
Totalmente & 40 & 60 \\
\hline
\end{tabular}

Fuente: Elaboración propia.

\section{Conclusiones}

La auditoría de gestión es un método de control que contribuye a lograr la eficiencia y la eficacia en el proceso de dirección estratégica de las instalaciones turísticas, ya que posibilita una retroalimentación oportuna con base en la detección de errores y sus correcciones para próximos periodos.

El plan de auditoría diseñado considera como área crítica el análisis estratégico dentro del proceso de dirección estratégica. Esta se evalúa considerando la elaboración de la misión y la visión, el diagnóstico y los objetivos estratégicos en cuanto a su contenido, diseño e implementación en el hotel Villa la Granjita.

La auditoría de gestión desarrollada tuvo como principales resultados:

- La elaboración de la misión fue evaluada como bueno, pues su contenido tuvo pocas cosas relevantes que mejorar y su diseño fue considerado como promedio.

- La confección de la visión fue calificada como bueno porque, a pesar de tener un contenido excelente, su diseño presenta algunos puntos que debe mejorar. 
- El diagnóstico estratégico tiene una calificación de inadecuado debido a que tanto su contenido como su diseño e implementación son inaceptables para el desarrollo del modelo de dirección estratégica.

- Los objetivos estratégicos son excelentes en razón de que su contenido y diseño son adecuados, aunque este resultado se ve afectado de manera indirecta y negativa por la evaluación del diagnóstico estratégico, ya que de este depende la pertinencia de los objetivos.

\section{Fuentes CONSULTADAS}

Gallego, J. (2007). Gestión de hoteles. Una nueva visión. Madrid: ThomsonParaninfo.

Heredia, M. y C. Martínez (2002). "Diagnóstico del ciclo administrativo. Caso práctico aplicado a una empresa”. Tesis de maestría. Santa Clara: Universidad Central "Marta Abreu" de Las Villas.

López, M. y D. García (2007). "La auditoría de gestión, una necesidad impostergable de la administración moderna”. Contribuciones a la economía [en línea]. Disponible en: http://www.eumed.net/ce/2007c/Auditoria \%20de\%20Gestion.htm [2013, 10 de abril].

Martínez, C. et al. (2007). "Metodología para el diagnóstico general con vistas al perfeccionamiento de la gestión en entidades turísticas". Ponencia presentada en la V Conferencia Internacional de Ciencias Empresariales, I Taller de Hotelería y Turismo. Santa Clara, Cuba.

Morales, C. (2007). "Procedimiento para el diseño de opciones turísticas en las agencias de viajes receptivo-minoristas de Villa Clara”. Tesis en opción al título de máster. Santa Clara: Universidad Central "Marta Abreu” de Las Villas.

Palominos, N. (2006). "Auditoría de gestión” [en línea]. Santiago: Universidad de Chile. Disponible en: http://vsoto2006.tripod.com/id2.html [2014, 3 de octubre]. 
Partido Comunista de Cuba (2011). "Lineamientos de la política económica y social del Partido y la Revolución” [en línea]. VI Congreso del Partido Comunista de Cuba. Disponible en: http://www.cubadebate.cu/ etiqueta/lineamientos-del-partido-y-la-revolucion/ [2011, 11 de abril].

Pérez, R. (2011). "Procedimiento para la utilización de la imagen como recurso estratégico en Villa la Granjita”. Tesis de maestría. Santa Clara: Universidad Central "Marta Abreu" de Las Villas.

Prieto, D. (2007). "Procedimiento para la implantación del cuadro de mando integral en pequeñas y medianas empresas hoteleras cubanas”. Tesis en opción al título de master. Santa Clara: Universidad Central "Marta Abreu" de Las Villas.

Stoner, J., E. Freeman y D. Gilbert (2006). Administración. 6a ed. México: Prentice Hall.

Suesta, V., J.A. Gutiérrez y J. Tornero (2001). “Comparativa de metodologías de diagnóstico empresarial” [en línea]. XVII Congreso Nacional de Ingeniería de Proyectos. Murcia. Disponible en: http//www.unizar.es/ aeipro/finder [2014, 3 de octubre]. 